\title{
Benchmark of thirteen bioinformatic pipelines for metagenomic virus diagnostics using datasets from clinical samples
}

Jutte J.C. de Vries $^{\text {a, }}$, Julianne R. Brown ${ }^{\mathrm{b}}$, Nicole Fischer ${ }^{\mathrm{d}}$, Igor A. Sidorov ${ }^{\mathrm{a}}$, Sofia Morfopoulou ${ }^{c}$, Jiabin Huang ${ }^{\mathrm{d}}$, Bas B. Oude Munnink ${ }^{\mathrm{e}}$, Arzu Sayiner ${ }^{\mathrm{f}}$, Alihan Bulgurcu ${ }^{g}$, Christophe Rodriguez $^{g}$, Guillaume Gricourt ${ }^{g}$, Els Keyaerts ${ }^{\text {h}}$, Leen Beller ${ }^{\text {h }}$, Claudia Bachofen ${ }^{\mathrm{i}}$, Jakub Kubacki $^{i}$, Cordey Samuel ${ }^{\mathrm{j}}$, Laubscher Florian ${ }^{\mathrm{j}}$, Schmitz Dennis ${ }^{\mathrm{k}}$, Martin Beer ${ }^{1}$, Dirk Hoeper $^{1}$, Michael Huber ${ }^{\mathrm{m}}$, Verena Kufner ${ }^{\mathrm{m}}$, Maryam Zaheri ${ }^{\mathrm{m}}$, Aitana Lebrand ${ }^{\mathrm{n}}$, Anna Papa ${ }^{\circ}$, Sander van Boheemen ${ }^{\mathrm{e}}$, Aloys C.M. Kroes ${ }^{\mathrm{a}}$, Judith Breuer ${ }^{\mathrm{b}, \mathrm{c}}$, F. Xavier Lopez-Labrador ${ }^{\mathrm{p}, \mathrm{q}}$, Eric C.J. Claas ${ }^{\mathrm{a}}$

${ }^{a}$ Clinical Microbiological Laboratory, Department of Medical Microbiology, Leiden University Medical Center, Leiden, the Netherlands

${ }^{\mathrm{b}}$ Microbiology, Virology and Infection Prevention \& Control, Great Ormond Street Hospital for Children NHS Foundation Trust, London, United Kingdom

${ }^{\mathrm{c}}$ Division of Infection and Immunity, University College London, London, United Kingdom

${ }^{\mathrm{d}}$ University Medical Center Hamburg-Eppendorf, UKE Institute for Medical Microbiology, Virology and Hygiene, Germany

e Viroscience, Erasmus Medical Center, Rotterdam, the Netherlands

${ }^{\mathrm{f}}$ Dokuz Eylul University, Medical Faculty, Izmir, Turkey

${ }^{\mathrm{g}}$ Hospital Henri Mondor, Paris, France

${ }^{\mathrm{h}}$ Laboratory of Clinical and Epidemiological Virology (Rega Institute), KU Leuven, Belgium

${ }^{\mathrm{i}}$ Institute of Virology, University of Zurich, Switzerland

${ }^{\mathrm{j}}$ Laboratory of Virology, University Hospitals of Geneva, Geneva, Switzerland

${ }^{\mathrm{k}}$ RIVM National Institute for Public Health and Environment, Bilthoven, the Netherlands

${ }^{1}$ Friedrich-Loeffler-Institute, Institute of Diagnostic Virology, Greifswald, Germany

${ }^{\mathrm{m}}$ Institute of Medical Virology, University of Zurich, Switzerland

${ }^{n}$ Swiss Institute of Bioinformatics, Geneva, Switzerland

${ }^{\circ}$ Department of Microbiology, Medical School, Aristotle University of Thessaloniki, Greece

${ }^{\mathrm{p}}$ Virology Laboratory, Genomics and Health Area, Center for Public Health Research (FISABIO-Public Health), Generalitat Valenciana and Microbiology \& Ecology Department, University of Valencia, Spain

${ }^{\mathrm{q}}$ CIBERESP, Instituto de Salud Carlos III, Spain

\section{A R T I C L E I N F O}

\section{Keywords:}

Viral metagenomics

Benchmark

Bioinformatic pipelines

\begin{abstract}
A B S T R A C T
Introduction: Metagenomic sequencing is increasingly being used in clinical settings for difficult to diagnose cases. The performance of viral metagenomic protocols relies to a large extent on the bioinformatic analysis. In this study, the European Society for Clinical Virology (ESCV) Network on NGS (ENNGS) initiated a benchmark of metagenomic pipelines currently used in clinical virological laboratories.

Methods: Metagenomic datasets from 13 clinical samples from patients with encephalitis or viral respiratory infections characterized by PCR were selected. The datasets were analyzed with 13 different pipelines currently used in virological diagnostic laboratories of participating ENNGS members. The pipelines and classification tools were: Centrifuge, DAMIAN, DIAMOND, DNASTAR, FEVIR, Genome Detective, Jovian, MetaMIC, MetaMix,
\end{abstract}

\footnotetext{
* Corresponding author.

E-mail addresses: jjcdevries@lumc.nl, jjcdevries@lumc.nl (J.J.C. de Vries), julianne.brown@gosh.nhs.uk (J.R. Brown), nfischer@uke.de (N. Fischer), I.A.

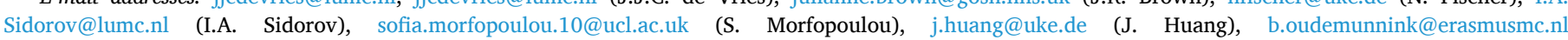

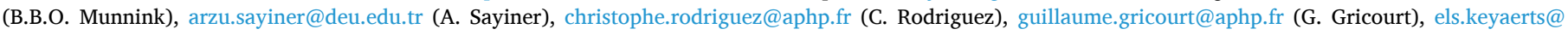

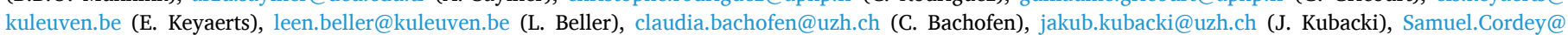

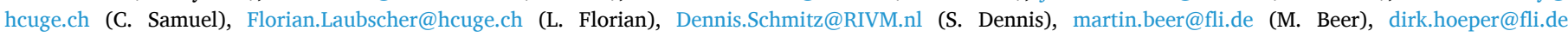

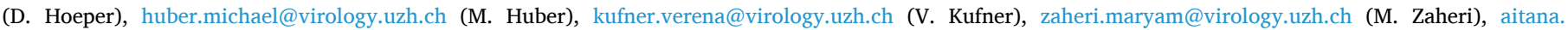

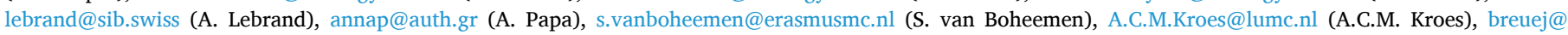
gosh.nhs.uk (J. Breuer), F.Xavier.Lopez@uv.es (F.X. Lopez-Labrador), E.C.J.Claas@lumc.nl (E.C.J. Claas).
} 
One Codex, RIEMS, VirMet, and Taxonomer. Performance, characteristics, clinical use, and user-friendliness of these pipelines were analyzed.

Results: Overall, viral pathogens with high loads were detected by all the evaluated metagenomic pipelines. In contrast, lower abundance pathogens and mixed infections were only detected by 3/13 pipelines, namely DNASTAR, FEVIR, and MetaMix. Overall sensitivity ranged from $80 \%$ (10/13) to 100\% (13/13 datasets). Overall positive predictive value ranged from $71-100 \%$. The majority of the pipelines classified sequences based on nucleotide similarity (8/13), only a minority used amino acid similarity, and 6 of the 13 pipelines assembled sequences de novo. No clear differences in performance were detected that correlated with these classification approaches. Read counts of target viruses varied between the pipelines over a range of 2-3 log, indicating differences in limit of detection.

Conclusion: A wide variety of viral metagenomic pipelines is currently used in the participating clinical diagnostic laboratories. Detection of low abundant viral pathogens and mixed infections remains a challenge, implicating the need for standardization and validation of metagenomic analysis for clinical diagnostic use. Future studies should address the selective effects due to the choice of different reference viral databases.

\section{Introduction}

Viral metagenomic next-generation sequencing (mNGS) is increasingly being used in virology laboratories for the diagnosis of patients with suspected but unexplained infectious diseases. The current main clinical application of viral metagenomics is for diagnosing encephalitis of unknown cause [1,2], but metagenomic sequencing is considered useful in a growing number of other clinical syndromes [3-6]. Although many wet-lab challenges need to be faced as well [14], the performance of metagenomic methods is largely dependent on accurate bioinformatic analysis, and both classification algorithms and databases are crucial factors determining the overall performance of the pipelines [7,55]. A wide range of metagenomic pipelines and taxonomic classifiers have been developed, commonly for the purpose of biodiversity studies analyzing the composition of the microbiome in different cohorts. In contrast, when applying metagenomics to patient diagnostics, potential false-negative and false-positive bioinformatic classification results can have significant consequences for patient care. Most reports on bioinformatic tools for metagenomic analysis for virus diagnostics typically describe algorithms and validations of single in-house developed pipelines developed by the authors themselves [8-12]. Most reports on bioinformatic tools for metagenomic analysis for virus diagnostics typically describe algorithms and validations of single in-house developed pipelines developed by the authors themselves [13], and recently a metagenomic benchmarking trial among Swiss virology laboratories has been conducted [7]. Recently, ESCV Network on NGS (ENNGS) recommendations for the introduction of next-generation sequencing in clinical virology, part II: bioinformatic analysis and reporting were published [55], aiming to address the challenges involved. While a professional External Quality Assessment (EQA) program is currently in preparation by Quality Control for Molecular Diagnostics (QCMD), the ENNGS [14,55] conducted the presented benchmark of bioinformatic pipelines of the participating diagnostic laboratories using viral metagenomic datasets derived from clinical samples, in order to assist laboratories with selection and optimization of tools to be implemented for clinical use.

\section{Methods}

\subsection{Datasets}

To exclude differences in wet-lab procedures, the same raw, untrimmed metagenomic datasets were provided, so that the participants had standardized datasets for bioinformatic analysis.

In total, 13 clinical metagenomic datasets from samples wellcharacterized by RT-PCR [15-18] were selected from patients with encephalitis or respiratory complaints, including: cerebrospinal fluid (CSF, $\mathrm{n}=4)$, brain biopsies $(\mathrm{n}=3)$, nasopharyngeal swabs $(\mathrm{n}=3)$, nasal washings $(\mathrm{n}=1)$, bronchoalveolar lavage $(\mathrm{n}=1)$, and a plasma sample $(\mathrm{n}=1)$. RT-PCR panel results and Cq-values are included in the result section. The pathogens in the 13 datasets are depicted in Table 2.

For samples processed at the Great Ormond Street Hospital, London (GOSH), mRNA from the three brain biopsy samples was sequenced on an Illumina NextSeq500 instrument using an 81 bp paired-run after library preparation using Illumina's TruSeq Stranded mRNA LT sample preparation kit ( $\mathrm{p} / \mathrm{n}$ RS-122-2101) according to the manufacturer's instructions [19]. The other samples were spiked with Equine Arteritis Virus (EAV) and Phocid Herpes Virus (PhHV) internal controls preceding total nucleic acid extraction using the MagNAPure 96 DNA and Viral NA Small Volume Kit (Roche Diagnostics, Almere, the Netherlands) and sequenced on Illumina NextSeq500 (respiratory samples) or NovaSeq6000 (CSF samples, plasma) instruments using 150 bp paired-end runs after library preparation using New England BioLabs' NEBNext Ultra Directional RNA Library preparation kit for Illumina with in-house adaptations in order to enable simultaneous detection of both DNA and RNA viruses, at the Leiden University Medical Center (LUMC) $[4,20]$. Three of the CSF samples were sequenced after enrichment using capture probes targeting vertebrate viruses [21]. Human reads from the output FASTQ files were removed after mapping them to human reference genome GRCh38 [22] with Bowtie2 version 2.3.4 [23] before the datasets were uploaded to various data sharing platforms (see below).

\subsection{Data sharing}

The FASTQ datasets were and remain publicly available for userfriendly downloading at https://veb.lumc.nl/CliniMG (hosted by the dept. MM, LUMC, Leiden), and part of the datasets were additionally accessible via a COMPARE Data Hub at http://www.ebi.ac.uk/ena/path ogens (hosted by the European Bioinformatics Institute, EMBL-EBI) [24].

\subsection{Bioinformatic pipelines}

The datasets were analysed in a blinded fashion by the participants, with the (viral) metagenomic pipelines and classification tools (Fig. 1 and Table 1) used at their diagnostic laboratories: Centrifuge [25], DAMIAN [26,27], DIAMOND [28], DNASTAR [29], FEVIR [30], Genome Detective [31], Jovian [32], MetaMIC [33], MetaMix [34,35], One Codex [36], RIEMS [37,38], Taxonomer [39], and VirMet [40]. DAMIAN was run by two participants in combination with a different database (pipeline A and B), and one participant run both Centrifuge and GenomeDetective. Details of the algorithms are described in Table 1.

\subsection{Performance characteristics}

Both qualitative and quantitative performance of the pipelines were analysed with real-time PCR results as gold standard. The following parameters available for all pipelines were considered: pathogen detection, taxonomic classification level and target read count. Additionally, horizontal genome coverage (if available), computational time, 
user-friendliness and output formats were considered. Since EAV and $\mathrm{PhHV}$ were added as internal controls and not reported by the participants (due to default reporting criteria, or absence in the database)they were not included in the comparative analysis.

\section{Results}

\subsection{Metagenomic pipeline characteristics}

In total 13 different metagenomic pipelines and classification tools were in use in the 13 participating diagnostic laboratories. Clinical use, classification and output characteristics of the pipelines and tools utilized are shown in Fig. 1 and Table 1. The majority of the pipelines were developed or adapted at a local site, while four pipelines were commercially available and web-based: DNASTAR (Madison, WI, USA), Genome Detective (Emweb bv, Herent, Belgium), One Codex (San Francisco, USA), and Taxonomer (Utah, USA). DAMIAN and Centrifuge are publicly available as an open source software. Both classification tools and reference databases differed among participants (and were fixed for end-users of the commercially available pipelines); (adapted versions of) NCBI's nucleotide and RefSeq databases were most commonly used to generate reference databases. Six of the 13 pipelines assembled sequence reads de novo, whereas the others classified unassembled reads. The majority of the pipelines classified reads based on nucleotide similarity $(8 / 13)$, and a minority used amino acid similarity $(2 / 13)$, or a combination of both (3/13 pipelines). Parameters used by the participants for defining a positive result were the number of virus reads, horizontal genome coverage (some of the participants), and a cutoff based on posterior-probability scores of the species presence (MetaMix) and ROC-curves. Output formats varied, the majority had a userfriendly output format: excel, PDF or interactive webpage. Examples of these user-friendly output formats are shown in Supplementary
Figure S1.

\subsection{Detection of PCR targeted viral pathogens; sensitivity}

The qualitative and quantitative results of the pipeline benchmarking for viruses detected by RT-PCR are shown in Table 2 and Fig. 2. Overall, higher abundance viral pathogens (Cq-value $<28)$ were detected by all metagenomic pipelines evaluated. In contrast, viral pathogens with RT-PCR Cq-value of 28 and higher including mixed virus infections were only detected by $3 / 13$ pipelines, namely DNASTAR, FEVIR, and MetaMix. Although participants analysed the same FASTQ files, read counts of the target viruses varied from one to several orders of magnitude across pipelines. Also, read counts (all datasets combined) achieved by participants did not correlate well with the viral load as measured by RT-PCR ( $R=-0.07$, P-value 0.5 ), however it must be noted that wet lab procedures varied per set of samples, including protocols with and without viral enrichment, which had potential impact on the viral read counts and thus on correlation with Cq-values. Overall sensitivity of the pipelines at sample level was $77 \%$ (10/13) - 100\% (13/ 13 samples, mixed infections counted as one) (Table 2 and Supplementary Table 2). At viral mNGS hit level, overall sensitivity was $80 \%$ $(12 / 15)-100 \%$ (15/15 viral hits) (Supplementary Table 4). One of the participants reported normalized reads including the genome length, using the following formula: RPKM $=$ (number of reads mapped to virus genome $\mathrm{Y} * 10^{6}$ ) / (total number of reads * length of genome in kp). This formula was also used to normalize the reads of all study pipelines shown in Fig. 2.

\subsection{Taxonomic level of classification}

The taxonomic levels of classification and typing of pathogenic viruses by the metagenomic pipelines with the settings used and reported
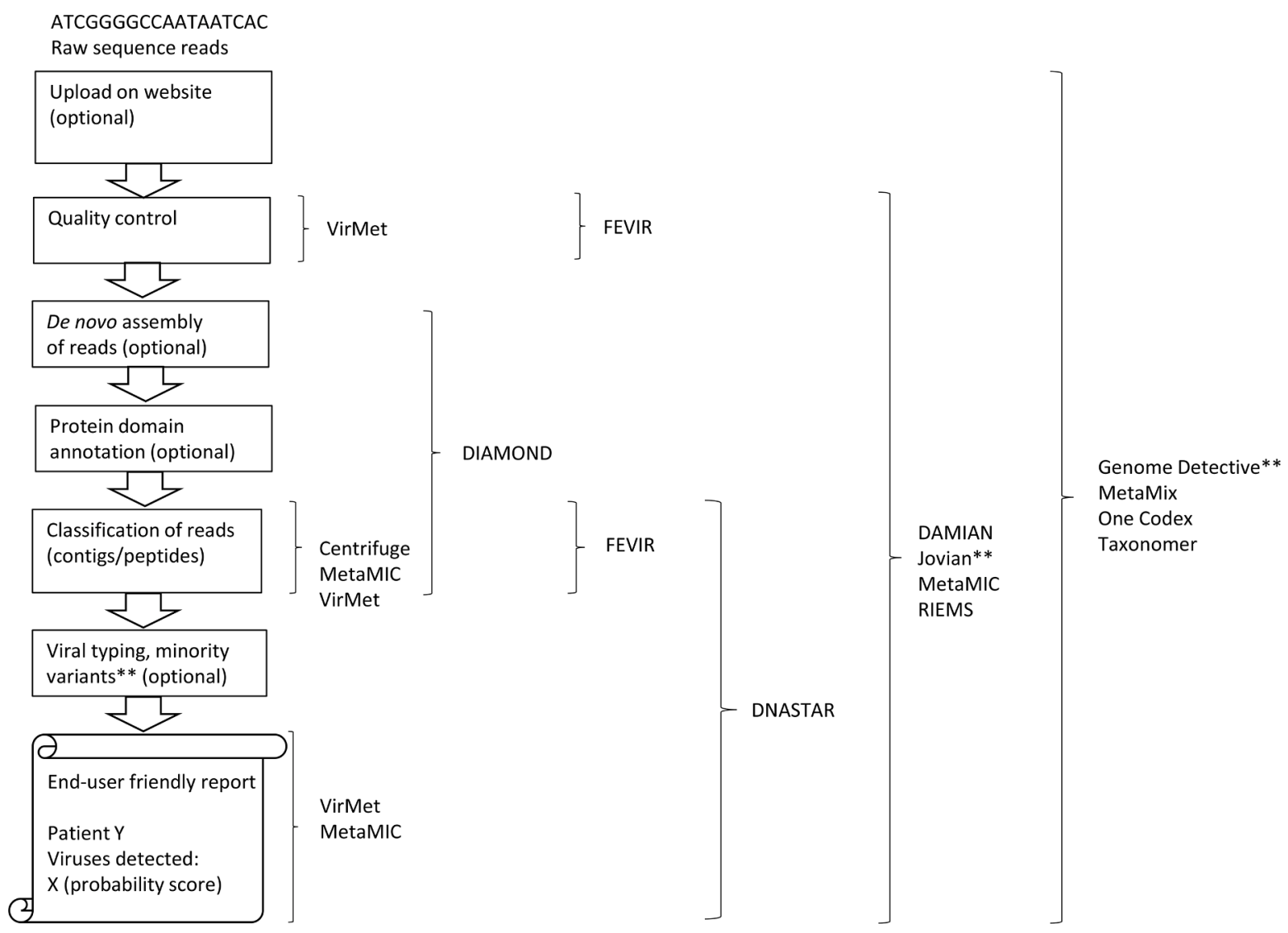

Fig. 1. Workflow of bioinformatic analysis of (viral) metagenomics data with the pipelines and classification tools used by participants in the current study. 
Table 1

Clinical use, classification and output characteristics of metagenomic pipelines analysed.

\begin{tabular}{|c|c|c|c|c|c|c|c|c|c|c|c|c|c|}
\hline $\begin{array}{l}\text { Pipeline no } \\
\text { (alphabetical } \\
\text { order) }\end{array}$ & 1 & 2 & 3 and $3 A$ & 4 & 5 & 6 & 7 & $8 \mathrm{ac}$ & 9 & 10 & 11 & 12 & 13 \\
\hline $\begin{array}{l}\text { Classification tool } \\
\text { or pipeline }\end{array}$ & $\begin{array}{l}\text { Centrifuge } \\
\text { [25] } \\
\text { v1.0.1-beta }\end{array}$ & $\begin{array}{l}\text { DAMIAN } \\
{[26,27]} \\
\text { v190628 }\end{array}$ & $\begin{array}{l}\text { DIAMOND } \\
{[28]} \\
\text { v0.9.13.114 }\end{array}$ & $\begin{array}{l}\text { DNASTAR } \\
{[29]} \\
\text { Lasergene } \\
\text { v16 }\end{array}$ & $\begin{array}{l}\text { FEVIR [30] } \\
\text { V1 }\end{array}$ & $\begin{array}{l}\text { Genome } \\
\text { Detective } \\
{[31] \text { v1.110 }}\end{array}$ & $\begin{array}{l}\text { Jovian [32] } \\
\text { Vo.9.6 }\end{array}$ & $\begin{array}{l}\text { MetaMIC } \\
\text { [33] v2.1.1 }\end{array}$ & $\begin{array}{l}\text { MetaMix } \\
\text { [34] [35] v1.2 }\end{array}$ & $\begin{array}{l}\text { One Codex } \\
\text { [36] v1 }\end{array}$ & $\begin{array}{l}\text { RIEMS } \\
{[37,38]} \\
\text { v4.0 }\end{array}$ & $\begin{array}{l}\text { Taxonomer } \\
{[39] 2020-\mathrm{U}}\end{array}$ & $\begin{array}{l}\text { VirMet [40] } \\
\text { v1.1.1 }\end{array}$ \\
\hline $\begin{array}{l}\text { Clinical usage by } \\
\text { participant }\end{array}$ & Patient care & $\begin{array}{l}\text { Experimen- } \\
\text { tal }\end{array}$ & Experimen-tal & Patient care & Patient care & Patient care ${ }^{\mathrm{a}}$ & Patient care & Patient care ${ }^{\mathrm{a}}$ & Patient care ${ }^{\mathrm{a}}$ & $\begin{array}{l}\text { Experimen- } \\
\text { tal }\end{array}$ & $\begin{array}{l}\text { Patient } \\
\text { care }\end{array}$ & $\begin{array}{l}\text { Experimen- } \\
\text { tal }\end{array}$ & Patient care \\
\hline $\begin{array}{l}\text { In-house/ } \\
\text { commercially } \\
\text { available }\end{array}$ & $\begin{array}{l}\text { Open-source } \\
\text { software }\end{array}$ & $\begin{array}{l}\text { Open-source } \\
\text { software }\end{array}$ & In-house & Commercial & In-house & Commercial & In-house & In-house & In-house & Commercial & In-house & Commercial & In-house \\
\hline Local/web-based & Local & Local & Local & $\begin{array}{l}\text { Local (cloud } \\
\text { optional) }\end{array}$ & Local & Web-based & Local & Local & $\begin{array}{l}\text { Web-based } \\
\text { (hosted on } \\
\text { Bluebee) }\end{array}$ & Web-based & Local & Web-based & Local \\
\hline De novo assembly & $\mathrm{N}$ & $\mathrm{Y}$ & $\mathrm{Y}[45]$ & $\mathrm{N}$ (optional) & $\mathrm{N}$ & $\mathrm{Y}$ & $\mathrm{Y}$ & $\mathrm{Y}$ & $\mathrm{Y}$ & $\mathrm{N}$ & $\mathrm{Y}$ & $\mathrm{N}$ & $\mathrm{N}$ \\
\hline $\begin{array}{l}\text { Alignment of NT/ } \\
\text { AA }\end{array}$ & NT & NT, AA & $\mathrm{AA}$ & NT & NT & $\mathrm{NT}, \mathrm{AA}$ & NT & NT & AA & NT & NT & NT, AA & NT \\
\hline $\begin{array}{l}\text { Database used by } \\
\text { participant } \\
\text { viral/bacterial } \\
\text { (version) }\end{array}$ & $\begin{array}{l}\text { Viruses, } \\
\text { bacteria, } \\
\text { fungi, archae; } \\
\text { RefSeq } \\
\text { (compressed } \\
\text { index) } \\
\text { V2019-04-04 }\end{array}$ & $\begin{array}{l}\text { NCBI's nt } \\
\text { and nr } \\
\text { v2019-05- } \\
17, \text { PFAM } \\
30.0\end{array}$ & $\begin{array}{l}\text { Viruses; NCBI's } \\
\text { non-redundant } \\
\text { protein } \\
\text { database/ } \\
\text { pipeline 3B: } \\
\text { NCBI's nt } \\
\text { V0.9.22 }\end{array}$ & $\begin{array}{l}\text { Based on } \\
\text { NCBI's nt } \\
\text { V2020-01- } \\
08\end{array}$ & $\begin{array}{l}\text { Viruses; based } \\
\text { on Virosaurus } \\
\text { [46] } \\
\text { v90v_2018_11 }\end{array}$ & $\begin{array}{l}\text { Viruses; } \\
\text { based on } \\
\text { RefSeq } \\
\text { (filtering: } \\
\text { Swissprot } \\
\text { Uniref 90) } \\
\text { v2018-11-14 }\end{array}$ & $\begin{array}{l}\text { NCBI's nt, } \\
\text { v2019-11-30 a. } \\
\text { o. (compressed } \\
\text { index) }\end{array}$ & $\begin{array}{l}\text { Bacteria, } \\
\text { viruses, fungi; } \\
\text { v2.1.1 } \\
\text { based on NCBI's } \\
\text { nt (complete) }\end{array}$ & $\begin{array}{l}\text { Human, } \\
\text { Environmental, } \\
\text { bacteria, Viruses } \\
\text { RefSeq protein } \\
\text { v2017 }\end{array}$ & $\begin{array}{l}\text { Bacteria, } \\
\text { viruses, fungi, } \\
\text { archaea, } \\
\text { protozoa, One } \\
\text { Codex DB } \\
\text { v2019-5-1 }\end{array}$ & $\begin{array}{l}\text { NCBI's nt } \\
\text { (complete) } \\
\text { v2019-3-16 }\end{array}$ & $\begin{array}{l}\text { Based on } \\
\text { NCBI's nt (v } \\
\text { May 2019) }\end{array}$ & $\begin{array}{l}\text { Based on NCBI's } \\
\text { nt (selection of } \\
\text { viral full } \\
\text { genomes without } \\
\text { compression) } \\
\text { v224 }\end{array}$ \\
\hline $\begin{array}{l}\text { Paired reads as } \\
\text { input option }\end{array}$ & $\mathrm{Y}$ & $\mathrm{Y}$ & Y & $\mathrm{Y}$ & $\mathrm{Y}$ & $\mathrm{Y}$ & $\mathrm{Y}$ & $\mathrm{Y}$ & $\mathrm{Y}$ & $\mathrm{Y}$ & $\mathrm{Y}$ & $\mathrm{N}$ & $\mathrm{N}$ \\
\hline $\begin{array}{l}\text { Trimming and } \mathrm{QC} \\
\text { tools }\end{array}$ & $\begin{array}{l}\text { Trimmo- } \\
\text { matic }\end{array}$ & $\begin{array}{l}\text { Trimmo- } \\
\text { matic }\end{array}$ & $\begin{array}{l}\text { Trimmo-matic } \\
\text { [47] }\end{array}$ & $\begin{array}{l}\text { Included in } \\
\text { DNASTAR }\end{array}$ & $\begin{array}{l}\text { Included in } \\
\text { virusscan [48] } \\
1.0\end{array}$ & & $\begin{array}{l}\text { Trimmomatic, } \\
\text { fastqc, multiqc }\end{array}$ & HMN Trimmer & $\begin{array}{l}\text { TrimGalore!, } \\
\text { prinseq }\end{array}$ & Cutadapt & $\begin{array}{l}\text { Roche } 454 \\
\text { newbler }\end{array}$ & $\mathrm{N}$ & Seqtk, prinseq \\
\hline $\begin{array}{l}\text { Exclusion of } \\
\text { human reads }\end{array}$ & $\mathrm{N}$ & $\mathrm{Y}$ & $\begin{array}{l}\text { N/pipeline 3B: } \\
\mathrm{Y}^{\mathrm{b}}\end{array}$ & $\mathrm{Y}$ & $\mathrm{N}$ & $\mathrm{Y}$ & $\mathrm{Y}$ & $\mathrm{Y}$ & $\mathrm{Y}$ & $\mathrm{Y}$ & $\mathrm{N}$ & $\mathrm{N}$ & $\mathrm{Y}$ \\
\hline Output type & Script, Krona & Excel & Script, krona & $\begin{array}{l}\text { User- } \\
\text { friendly }\end{array}$ & Script & $\begin{array}{l}\text { Web interface, } \\
\text { interactive }\end{array}$ & $\begin{array}{l}\text { Web interface, } \\
\text { interactive }\end{array}$ & PDF & $\begin{array}{l}\text { Web interface, } \\
\text { interactive, PDF } \\
\text { and excel }\end{array}$ & $\begin{array}{l}\text { Web } \\
\text { interface, } \\
\text { interactive, } \\
\text { PDF and excel }\end{array}$ & PDF & $\begin{array}{l}\text { Web } \\
\text { interface, } \\
\text { interactive }\end{array}$ & PDF \\
\hline $\begin{array}{l}\text { Visualization of } \\
\text { genome } \\
\text { coverage }\end{array}$ & $\mathrm{N}$ & $\mathrm{N}$ & $\mathrm{N}$ & $\mathrm{Y}$ & $\mathrm{N}$ & $\mathrm{Y}$ & $\mathrm{N}$ & $\mathrm{N}$ & $\begin{array}{l}\mathrm{N} \\
\text { (CLC Genomics } \\
\text { Workbench) }\end{array}$ & $\mathrm{N}$ & $\mathrm{Y}$ & $\begin{array}{l}\mathrm{N} \text { (free } \\
\text { version)/ } \mathrm{Y} \\
\text { (paid) }\end{array}$ & $\mathrm{Y}$ \\
\hline $\begin{array}{l}\text { Computational } \\
\text { time for } \\
\text { analysis per } \\
\text { sample (CPU/ } \\
\text { RAM) }\end{array}$ & $\begin{array}{l}\sim 10 \mathrm{~nm}(24 \\
\text { CPUs, } 0.3 \mathrm{~GB} \\
\text { RAM) }\end{array}$ & $\begin{array}{l}90 \mathrm{~nm}(56 \\
\text { CPUs, } 125 \\
\text { GB RAM) }\end{array}$ & $\begin{array}{l}\text { 90min ( } 36 \\
\text { CPUs, } \\
\text { RAM=23 gb) }\end{array}$ & $\begin{array}{l}3 \mathrm{~nm}(4 \\
\text { CPUs, } 64 \mathrm{~GB} \\
\text { RAM }\end{array}$ & $\begin{array}{l}1 \mathrm{~nm}(176 \\
\text { CPUs, } 384 \mathrm{~GB} \\
\text { RAM) }\end{array}$ & $\begin{array}{l}\sim 10 \mathrm{~nm} \text { (web- } \\
\text { based) }\end{array}$ & $\begin{array}{l}12 \mathrm{~nm} \text { ( } 20 \text { CPUs, } \\
\text { U GB RAM) }\end{array}$ & $\begin{array}{l}18 \text { nm (U CPUs, } \\
\text { U GB RAM) }\end{array}$ & $\begin{array}{l}\text { 60-180 nm (web- } \\
\text { based) }\end{array}$ & $\begin{array}{l}35-40 \mathrm{~nm} \\
\text { (web-based) }\end{array}$ & $\begin{array}{l}\text { U ( } 48 \\
\text { CPUs, } 768 \\
\text { GB RAM) }\end{array}$ & $\begin{array}{l}10 \mathrm{~nm} \text { (web- } \\
\text { based) }\end{array}$ & $\begin{array}{l}20 \text { nm (16 CPUs, } \\
64 \text { GB RAM) }\end{array}$ \\
\hline $\begin{array}{l}\text { Cut-off for } \\
\text { defining } \\
\text { positive result } \\
\text { used }\end{array}$ & $\begin{array}{l}\geq 15 \text { reads } \\
{[20]}\end{array}$ & $\begin{array}{l}\text { Contig } \\
\text { length }=> \\
400 \mathrm{bp}\end{array}$ & 1 read & 1 read & $\begin{array}{l}\geq 300 \mathrm{nt} \\
\text { coverage }\end{array}$ & $\begin{array}{l}\geq 3 \text { regions, } \\
\text { distributed } \\
\text { [49] }\end{array}$ & $\mathrm{U}$ & $\begin{array}{l}\text { Above } \\
\text { background: } \\
\text { environmental } \\
\text { sample }\end{array}$ & $\begin{array}{l}\geq 3 \text { regions } \\
>10 \text { reads }[50] \\
{[51]} \\
\text { Probability score }\end{array}$ & 1 read & 1 read & $1 \mathrm{read}$ & $\begin{array}{l}\geq 3 \text { reads, } \\
\text { distributed, } \\
>100 x \text { than } \mathrm{NC} / \\
\text { other samples }\end{array}$ \\
\hline $\begin{array}{l}\text { Confirmatory } \\
\text { analysis } \\
\text { required for } \\
\text { clinical } \\
\text { reporting }\end{array}$ & BLAST, PCR & $\begin{array}{l}\text { PCR for } \\
\text { clinical cases }\end{array}$ & $\begin{array}{l}\text { BLAST, PCR for } \\
\text { clinical cases }\end{array}$ & $\begin{array}{l}\text { BLAST, PCR } \\
\text { for clinical } \\
\text { cases }\end{array}$ & BLAST & $\mathrm{U}$ & $\mathrm{U}$ & $\begin{array}{l}\text { PCR not } \\
\text { required (based } \\
\text { on validation) } \\
{[52-54]}\end{array}$ & $\begin{array}{l}\text { BLAST, coverage } \\
\text { (PCR not required } \\
\text { based on } \\
\text { validation) }\end{array}$ & Not required & $\begin{array}{l}\text { BLAST, } \\
\text { PCR }\end{array}$ & BLAST & $\mathrm{U}$ \\
\hline
\end{tabular}

AA; amino acid, NT; nucleotide, U; Undisclosed.

${ }^{\text {a }}$ Within the scope of accreditation.

b Mapping of the trimmed reads to HG38 by Bowtie with "very-sensitive" option. 
Table 2

Qualitative and quantitative results: raw sequence read count categories of the PCR positive viruses reported by the metagenomic pipelines using datasets from 13 clinical samples, per classification tool (complete pipeline details can be found in Table 1). CSF; cerebrospinal fluid, NP; nasopharyngeal, BAL; bronchoalveolar lavage, and in legend: ND; not detected.

\section{XXX}

by the participants are shown in Fig. 3 and Supplementary Table 3 . The classification level is dependent on the database used, algorithm settings (classification of reads to the lowest common ancestor, LCA, in case of multiple hits), and the participant's default reporting levels based on either in-house validation data or clinical relevancy. Species level classification was the most common level reported. Serotype and strain level were identified by tools that were combined with NCBI's nt database without the LCA setting. DAMIAN was the only tool to report classification at the isolate level.

For the Adenovirus sample (\#13), virus types reported were not

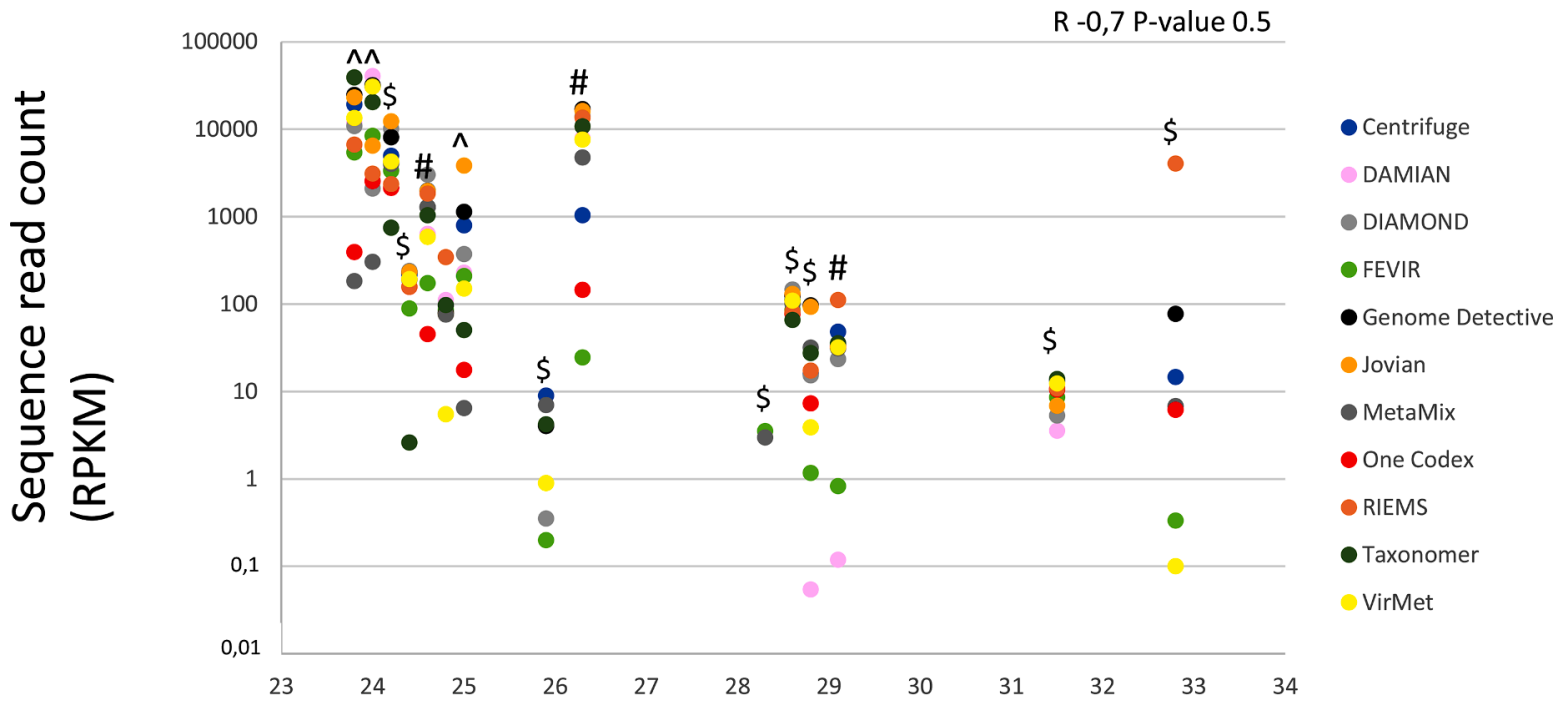

Cq value

Fig. 2. Sequence read counts (Y) versus RT-PCR Cq values (X) of the PCR positive viruses using datasets from 13 clinical samples, per classification tool with read counts reported by the participants (complete pipeline details can be found in table 1). Each vertical series of dots represents one clinical sample. The different wet lab methods used are marked ( $~$ mRNA sequencing, \$ RNA/DNA sequencing, and \#: a captured approach using probes targeting vertebrate viruses). RPKM; reads per kilobase of genome per million mapped reads.

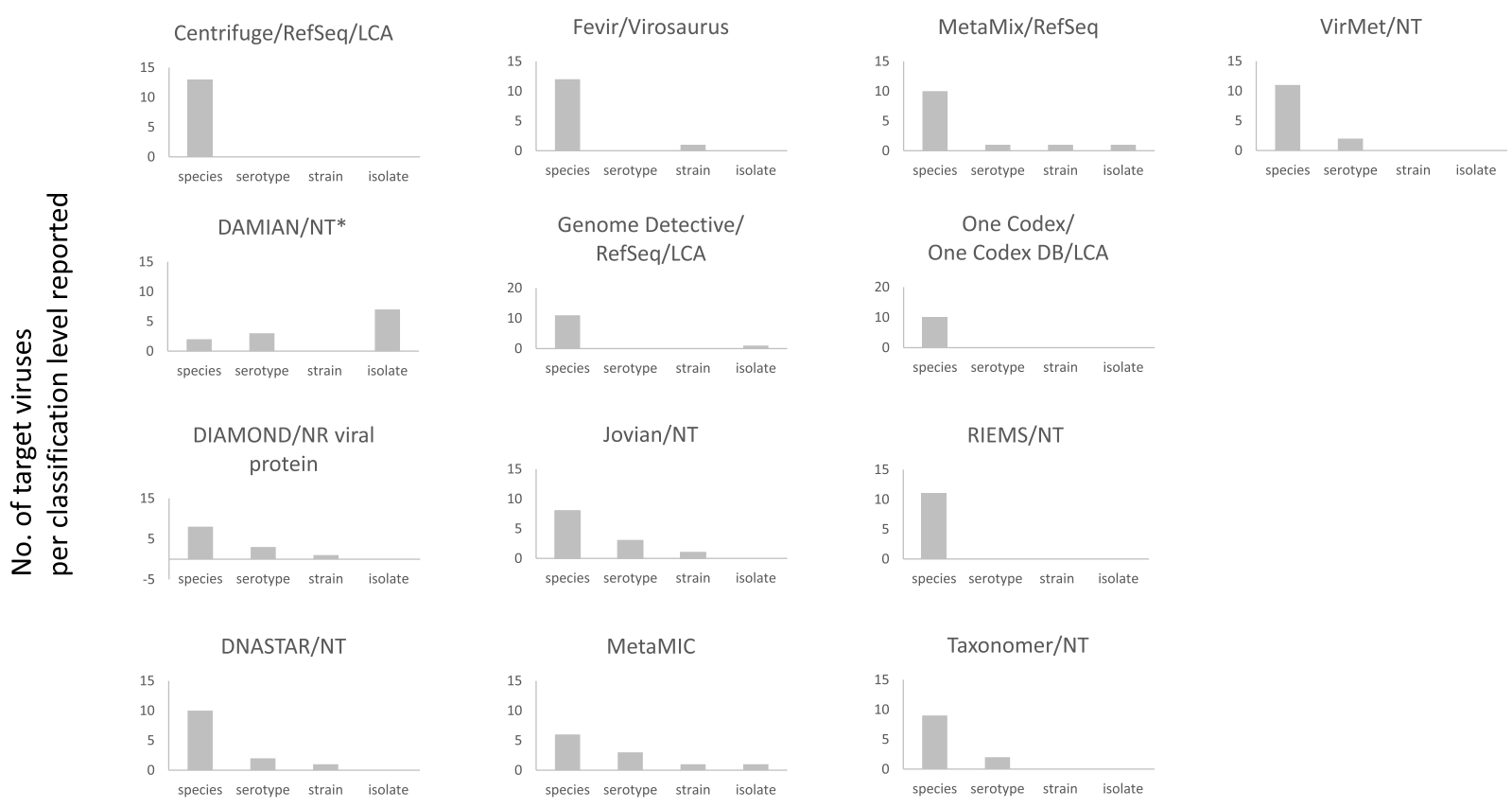

Fig. 3. (Taxonomic) level of classification and typing of the pathogenic viruses identified using the combination of tools and databases, as reported by participating diagnostic laboratories. Depicted are the number of target viruses per classification level.RefSeq; NCBI's RefSeq data base (or an adapted version), NT; NCBI's nucleotide database (or an adapted version); LCA; lowest common ancestor. *Taxonomic assignment method described in $[25,26]$ 
consistent between different pipelines: human Adenovirus type 31 (DIAMOND, Jovian, DNASTAR, VirMet), type 12 (DAMIAN), type 31 or 61 (metaMIC), indicating that type classification was not always correct. Type 12 and 31 are both from subgroup A Adenoviruses, whereas type 61 is a type 31 recombinant virus.

\subsection{Additional virus hits and positive predictive value}

Additional viruses, either not tested for by RT-PCR or RT-PCR negative were reported by 11 out of 13 pipelines, and in one or more samples (Supplementary Table 4). The following additional viruses were reported by multiple pipelines and absent in the negative run control (dataset not available for the participants): human retrovirus RD114 (2-2102 reads, up to $28 \%$ genome coverage), feline leukemia virus (2-1406 reads), torque-teno virus (TTV) (18-66 reads, up to $7 \%$ genome coverage), polyomaviruses (5-41 reads, up to $37 \%$ genome coverage), Bovine viral diarrhea virus (BVDV) (6-220 reads, likely FBS contaminants), human metapneumovirus (HMPV) (15-21 reads, 9\% genome coverage), human rhinovirus (HRV) (2-4 reads, up to $5 \%$ genome coverage), human parainfluenzavirus-4 (PIV-4) (2-6 reads) and Dengue virus (18-370 reads). RT-PCR data were available for some of the additional viruses detected (Supplementary Table 4). When considering viral mNGS hits with negative RT-PCR results: CoV-NL63 (1 read), PIV-4 (2-6 reads), HRV-C (2-4 reads), CoV-OC43 (5 reads), INF-B ( 2 reads), the positive predictive value ranged from $71-100 \%$ (Fig. 4). It must be noted that for these mNGS hits, no distinction could be made between assignments of sequences genuinely present e.g. by index hopping (which was suspected given the low number of reads), false negative by PCR due to primers/probes mismatches, and false positive assignments. When considering the mNGS findings without available RT-PCR results, retrovirus RD114, leukemia viruses, TTV, and polyomaviruses sequences may actually be present given their association with the host (integrated or commensal).

\subsection{Reporting criteria}

Reporting criteria used by the participants are shown in Table 1: a threshold for number of reads, for genome coverage (number of nucleotides and proportion of the genome, or a certain number of genome regions covered), based on reference or in-house validation studies. A BLAST analysis of matching sequences was commonly used by the study participants to exclude false positive (or to confirm true positive) hits. Some participants indicated that for clinical samples outside of the current benchmark, they required a confirmatory PCR before reporting while others indicated that this was not needed based on experiences from their validation studies.

\section{Discussion}

This study aimed to benchmark the combination of bioinformatic tools and databases currently in use in diagnostic virology laboratories from the ESCV ENNGS network. The data presented here support bioinformatic selection and optimization of software for the implementation of viral metagenomic sequencing for pathogen detection in clinical samples. To our knowledge, this is the first large-scale international benchmarking study using datasets from clinical samples and pipelines currently applied in a large series of clinical diagnostic laboratories.

The study showed that the pipelines of all the participating laboratories succeeded in detecting viral pathogens with relative high viral loads (Cq-values $<28$ ), whereas lower abundant pathogens and mixed infections were only detected by some of the pipelines, namely DNASTAR, FEVIR, and MetaMix. These results are in line with other reports [7]. With regard to mixed infections, the less abundant viruses were generally missed, possibly due to the low number of reads, or reporting considerations. For the missed CoV-HKU1 virus, potential primer cross-reactivity with CoV-NL63 viruses was excluded by in silico analysis. The databases used in the pipelines were mostly custom-made, based on either NCBI's RefSeq [41] or nt database [42]. All of the participants used different classification tools, though no selection of laboratories using different tools was made in advance. Given the inclusion of different types of pipelines including commercially available ones with fixed databases, it was not feasible to compare the different tools with one standardized database at the local sites. Two of the three pipelines that reached $100 \%$ sensitivity included NCBI's nt database but this was also seen using a pipeline with NCBI's RefSeq database. Pipelines with NCBI's nt database scored both low and maximum precision.

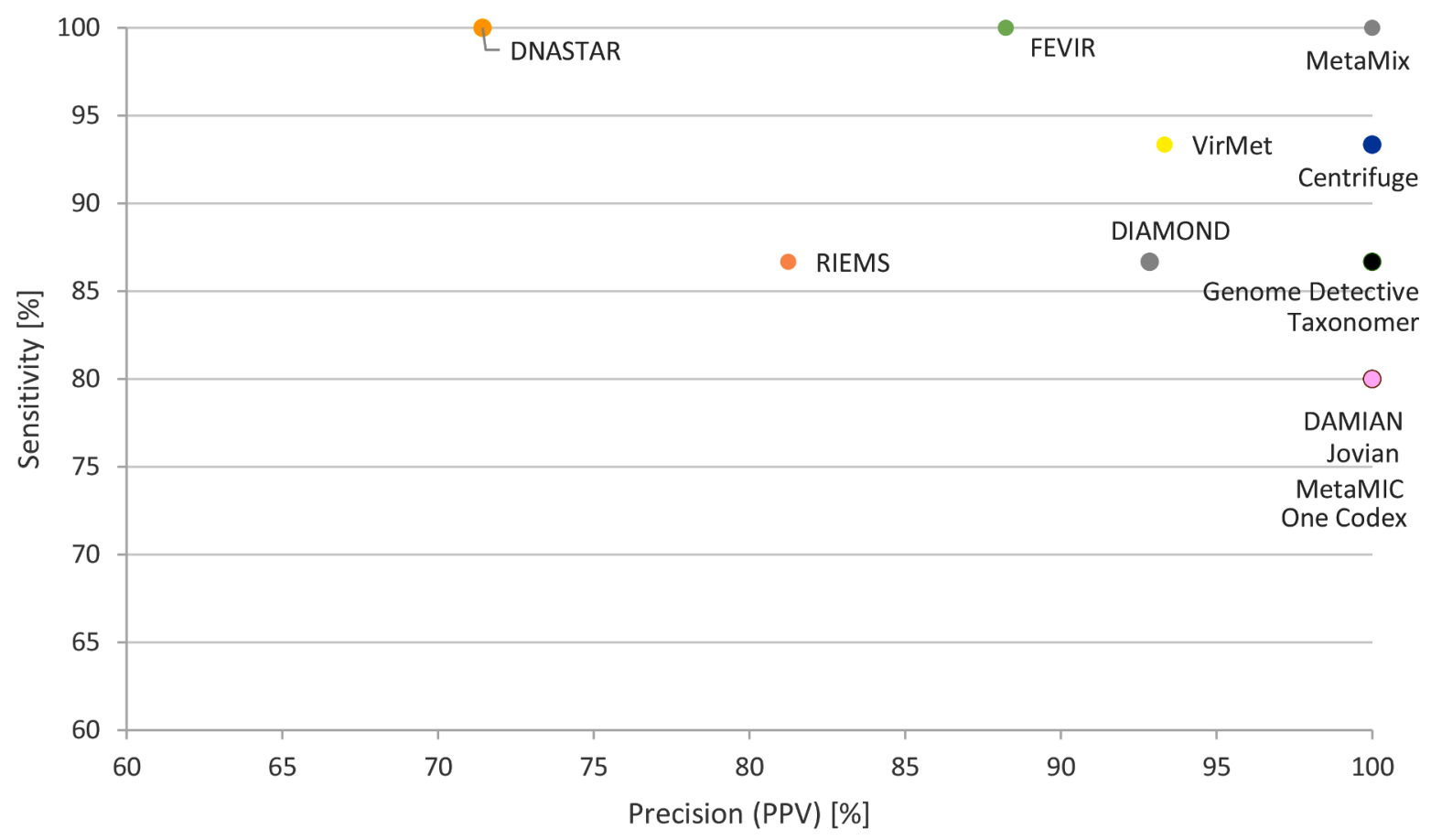

Fig. 4. Sensitivity and positive predictive value based on the hits reported by the participants, for the different pipelines/classification tools. 
The design did allow for comparison of the complete pipeline in use for clinical diagnostics, from QC to reporting algorithms including posterior probability scores. No clear differences were observed in terms of performance based on nucleotide-based classification versus amino acid-based classification and de novo assembly-based algorithms versus read based classification: whereas amino-acid based classification may be more sensitive for detecting variants, two of the three pipelines with $100 \%$ sensitivity used nucleotide-based classification (DNASTAR, FEVIR). High precision was reached by pipelines that used de novo assembly but this was not essential: $3 / 8$ pipelines with $100 \%$ precision did not use de novo assembly (Centrifuge, Taxonomer, One Codex).

Reported read counts and genome coverage varied between pipelines up to several orders of magnitude (for read counts), explaining in part the differences observed in limits of detection for samples with very low viral load. Possibly, differences in reporting of unique versus nonuniquely mapped sequence reads may be related to this difference. Sensitivity and positive predictive value were measured, conveniently avoiding the proportion of true negative findings given the immense but unknown number of negative mNGS hits without RT-PCR data needed for specificity calculations. This aspect remains a limitation intrinsically linked to mNGS validations with clinical datasets. Datasets from negative matrix samples would have been of use for specificity calculations. Positive predictive value calculations were hampered by the intrinsic inability to distinguish between sequences actually present in the dataset that might be undetected by RT-PCR because of, for instance, primer mismatches, index hopping or contaminant sequences introduced during library preparation. This may partially be overcome by defining mNGS consensus results as alternative golden standard, however in diagnostic settings e.g. index hopping reads should not be labeled positive despite being actually present in the dataset. A study design using synthetic data for example spiked into real-data mimicking reallife situations would enable accurate estimation of the specificity and PPV whilst taking into consideration interfering real-life factors such as sequences with switched indices and 'kitome' sequences, present in every single dataset. Future studies could address specificity analysis using artificial datasets that take into account the index hopping phenomenon.

It is important to note that participants likely have optimized their interpretation algorithm including cut-offs for their specific workflow from library preparation to sequencing. A different wet lab procedure (sequencer with or without index hopping, preparation with or without probe enrichment) will require new validation and indexing of the determined cut-off values and probability values. Because this was a dry lab comparison exercise, the participants could not follow their routine wet lab workflow and confirmatory PCR steps, which may have affected the reporting of results. Therefore no conclusions can be drawn on the limit of detection of the full metagenomic workflows used in each specific laboratory. The two labs that distributed the datasets may have been advantageous with regard to the analyses since the cut-offs for defining a positive result likely have been adjusted to the specific wet lab procedure used. However, the scores from the pipelines in use in these labs were not higher for the specific datasets delivered, in contrast, all pipelines detected all samples distributed by lab A, and other participants were more successful in detection some of the most challenging samples distributed by lab B.

Genome coverage and depth was not always taken into account by the participating laboratories, however can be an effective parameter to distinguish between (PCR-)contaminants, often indicated by high depth at a small (PCR amplicon) region of the genome, and true positives [21, 55]. In five of participating laboratories a cut-off of one single read was chosen for defining a positive mNGS result. While potentially at higher risk of reporting false positive results, the PPV of these pipelines ranged from 72 up to $100 \%$, indicating that this cut-off was dependent on the overall steps of the analysis and reporting. ROC analysis was used to find the optimal balance between sensitivity and specificity [20].

Finally, our taxonomic results are in line with data available from other groups [43]: the pipelines performed well at species level but deeper level classification was subject to less reliable classification in some cases.

In conclusion, a wide variety of viral metagenomic pipelines with overall high sensitivity are currently used in the ESCV ENNGS participating clinical diagnostic laboratories. Detection of low abundance viral pathogens or mixed infections remains a challenge, implicating the need for standardization and validation of metagenomic analysis for clinical diagnostic use [44]. The algorithm for defining positive results and rejecting false positive results is critical and should be evaluated individually for every workflow, which includes genome extraction, library preparation, sequencer and bioinformatic pipeline. Identification of deeper taxonomic levels is challenging, dependent on the individual types present in the reference database, and should be validated separately to prevent misidentification.

\section{Author contributions}

Conceptualization: JV, EC. Methodology: JV, EC, FXL, NF, IS, BO, SB. Formal analysis: JV, JuB, NF, IS, SM, JH, BO, AS, AB, CR, GG, EK, LB, CB, JK, SC, FL, DS, MB, DH, MH, VK, MZ, AL, AP. Visualization: JV, AL. Writing - original draft: JV. Writing - review \& editing: all authors.

\section{Declaration of Competing Interest}

No conflicts of interest.

\section{Acknowledgements}

We thank the COMPARE study group (https://www.compareeurope.eu/) and the EMBL-EBI (https://www.ebi.ac.uk/) for the availability of the Data Hubs.

Funding

MH was supported by the Clinical Research Priority Program 'Comprehensive Genomic Pathogen Detection' of the University of Zurich. FXL received funding from Instituto de Salud Carlos III, Spain (Grant numbers PI18/01824 and PI18/01759 and CIBEResp).

\section{Supplementary materials}

Supplementary material associated with this article can be found, in the online version, at doi:10.1016/j.jcv.2021.104908.

\section{References}

[1] J.R. Brown, T. Bharucha, J. Breuer, Encephalitis diagnosis using metagenomics: application of next generation sequencing for undiagnosed cases, J. Infect. 76 (3) (2018) 225-240.

[2] M.R. Wilson, et al., Clinical Metagenomic Sequencing for Diagnosis of Meningitis and Encephalitis, N. Engl. J. Med. 380 (24) (2019) 2327-2340.

[3] H. Jerome, et al., Metagenomic next-generation sequencing aids the diagnosis of viral infections in febrile returning travellers, J. Infect. 79 (4) (2019) 383-388.

[4] S. van Boheemen, et al., Retrospective validation of a metagenomic sequencing protocol for combined detection of RNA and DNA viruses using respiratory samples from pediatric patients, J. Mol. Diagn. 22 (2) (2020) 196-207.

[5] D.W. Lewandowska, et al., Metagenomic sequencing complements routine diagnostics in identifying viral pathogens in lung transplant recipients with unknown etiology of respiratory infection, PLoS One 12 (5) (2017), e0177340.

[6] V. Kufner, et al., Two years of viral metagenomics in a tertiary diagnostics unit: evaluation of the first 105 Cases, Genes (Basel) 10 (9) (2019).

[7] T. Junier, et al., Viral metagenomics in the clinical realm: lessons learned from a Swiss-wide ring trial, Genes (Basel) 10 (9) (2019).

[8] J. Chen, J. Huang, Y. Sun, TAR-VIR: a pipeline for TARgeted VIRal strain reconstruction from metagenomic data, BMC Bioinform. 20 (1) (2019) 305.

[9] S. Miller, et al., Laboratory validation of a clinical metagenomic sequencing assay for pathogen detection in cerebrospinal fluid, Genome Res. 29 (5) (2019) 831-842.

[10] D. Paez-Espino, et al., Nontargeted virus sequence discovery pipeline and virus clustering for metagenomic data, Nat. Protoc. 12 (8) (2017) 1673-1682.

[11] Y. Li, et al., VIP: an integrated pipeline for metagenomics of virus identification and discovery, Sci. Rep. 6 (2016) 23774. 
[12] S. Nooij, et al., Overview of virus metagenomic classification methods and their biological applications, Front. Microbiol. 9 (2018) 749.

[13] A. Brinkmann, et al., Proficiency testing of virus diagnostics based on bioinformatics analysis of simulated in silico high-throughput sequencing data sets J. Clin. Microbiol. 57 (8) (2019).

[14] F.X. Lopez-Labrador, Fischer N. B.J.R., H. Harvala, S. Van Boheemen, O Cinek, A Sayiner, T Vasehus Madsen, E. Auvinen, et al., Recommendations for the introduction of metagenomic high-throughput sequencing in clinical virology, part I: wet lab procedure, J. Clin. Virol. (2020). Dec.

[15] J.S. Kalpoe, et al., Validation of clinical application of cytomegalovirus plasma DNA load measurement and definition of treatment criteria by analysis of correlation to antigen detection, J. Clin. Microbiol. 42 (4) (2004) 1498-1504.

[16] S.J. Read, J.B. Kurtz, Laboratory diagnosis of common viral infections of the central nervous system by using a single multiplex PCR screening assay, J. Clin. Microbiol. 37 (5) (1999) 1352-1355.

[17] A.C. Lankester, et al., Epstein-Barr virus (EBV)-DNA quantification in pediatric allogenic stem cell recipients: prediction of EBV-associated lymphoproliferative disease, Blood 99 (7) (2002) 2630-2631.

[18] K. Loens, et al., Performance of different mono- and multiplex nucleic acid amplification tests on a multipathogen external quality assessment panel, J. Clin. Microbiol. 50 (3) (2012) 977-987.

[19] S. Morfopoulou, et al., Deep sequencing reveals persistence of cell-associated mumps vaccine virus in chronic encephalitis, Acta Neuropathol. 133 (1) (2017) $139-147$.

[20] A.L. van Rijn, et al., The respiratory virome and exacerbations in patients with chronic obstructive pulmonary disease, PLoS One 14 (10) (2019), e0223952.

[21] E.C. Carbo, et al., Improved diagnosis of viral encephalitis in adult and pediatric hematological patients using viral metagenomics, J. Clin. Virol. 130 (2020), 104566 https://www.ncbi.nlm.nih.gov/assembly/GCF_000001405.26/(Accessed July.

[22] S.L.S. B. Langmead, Fast gapped-read alignment with Bowtie 2, Nat. Methods and h.d.o.n 9 (4) (2012) 357-359. Apr.

[23] C. Amid, et al., The COMPARE data hubs, Database- J. Biol. Databases Curat. (2019) 1-14.

[24] D. Kim, et al., Centrifuge: rapid and sensitive classification of metagenomic sequences, Genome Res. 26 (12) (2016) 1721-1729.

[25] M. Alawi, et al., DAMIAN: an open source bioinformatics tool for fast, systematic and cohort based analysis of microorganisms in diagnostic samples, Sci. Rep. 9 (1) (2019) 16841, https://sourceforge.net/projects/damian-pd.

[26] B. Buchfink, C. Xie, D.H. Huson, Fast and sensitive protein alignment using DIAMOND, Nat. Methods 12 (1) (2015) 59-60, https://www.dnastar.com/ software/lasergene/.

[27] J.F. Fernandes, et al., Unbiased metagenomic next-generation sequencing of blood from hospitalized febrile children in Gabon, Emerg. Microbes. Infect. 9 (1) (2020) $1242-1244$.

[28] M. Vilsker, et al., Genome detective: an automated system for virus identification from high-throughput sequencing data, Bioinformatics 35 (5) (2019) 871-873, www.genomedetective.com.https://github.com/DennisSchmitz/Jovian.
[29] C. Rodriguez, et al., Pathogen identification by shotgun metagenomics of patients with necrotizing soft-tissue infections, Br. J. Dermatol. (2019).

[30] S. Morfopoulou, V. Plagnol, Bayesian mixture analysis for metagenomic community profiling, Bioinformatics 31 (18) (2015) 2930-2938, https://cran.rproject.org/web/packages/metaMix/index.html.

[31] S.S. Minot, One Codex: a sensitive and accurate data platform for genomic microbial identification, bioRxiv (2015).

[32] M. Scheuch, D. Hoper, M. Beer, RIEMS: a software pipeline for sensitive and comprehensive taxonomic classification of reads from metagenomics datasets, BMC Bioinform. 16 (2015) 69, https://github.com/EBI-COMMUNITY/fli-RIEMS.

[33] S. Flygare, et al., Taxonomer: an interactive metagenomics analysis portal for universal pathogen detection and host mRNA expression profiling, Genome Biol, 17 (1) (2016) 111, https://github.com/medvir/VirMet and https://github.com/ medvir/shiny-server/tree/master/NGS/VirMetRunAnalysis.

[34] N.A. O'Leary, et al., Reference sequence (RefSeq) database at NCBI: current status, taxonomic expansion, and functional annotation, Nucleic. Acids. Res. 44 (D1) (2016) D733-D745.

[35] D.A. Benson, et al., GenBank, Nucleic Acids Res. 39 (2011) D32-D37. Database issue.

[36] A. Sczyrba, et al., Critical assessment of metagenome interpretation-a benchmark of metagenomics software, Nat. Methods 14 (11) (2017) 1063-1071.

[37] T. Bharucha, et al., STROBE-metagenomics: a STROBE extension statement to guide the reporting of metagenomics studies, Lancet Infect. Dis. 20 (10) (2020) e251-e260.

[38] S. Nurk, et al., metaSPAdes: a new versatile metagenomic assembler, Genome Res. 27 (5) (2017) 824-834, https://viralzone.expasy.org/8676.

[39] A.M. Bolger, M. Lohse, B. Usadel, Trimmomatic: a flexible trimmer for Illumina sequence data, Bioinformatics 30 (15) (2014) 2114-2120, https://github.com/sibswiss/virusscan.

[40] E.C Carbo, Karelioti E. B.E., I. Sidorov, M.C.W. Feltkamp, P.A. Von dem Borne, J.G. M. Verschuuren Jan, A.C.M. Kroes, E.C.J. Claas, J.J.C. De Vries, Improved diagnosis of viral encephalitis in adults and pediatric hematological patients using viral metagenomics, bioRxiv (2020).

[41] K. Mongkolrattanothai, J.D. Bard, The utility of direct specimen detection by Sanger sequencing in hospitalized pediatric patients, Diagn. Microbiol. Infect. Dis. 87 (2) (2017) 100-102.

[42] J. Kawada, et al., Identification of viruses in cases of pediatric acute encephalitis and encephalopathy using next-generation sequencing, Sci. Rep. 6 (2016) 33452.

[43] C. Rodriguez, et al., Pathogen identification by shotgun metagenomics of patients with necrotizing soft-tissue infections, Br. J. Dermatol. 183 (1) (2020) 105-113.

[44] C. Rodriguez, et al., Fatal measles inclusion-body encephalitis in adult with untreated AIDS, France, Emerg. Infect. Dis. 26 (9) (2020) 2231-2234.

[45] C. Rodriguez, et al., Fatal encephalitis caused by cristoli virus, an emerging orthobunyavirus, France, Emerg. Infect. Dis. 26 (6) (2020) 1287-1290.

[46] J.J.C. De Vries, et al., Recommendations for the introduction of next-generation sequencing in clinical virology, part II: bioinformatic analysis and reporting, J. Clin. Virol. (2021), 104812, https://doi.org/10.1016/j.jcv.2021.104812 https:// doi.org/. 